\title{
The Design and Implementation of a Certain Type of Job Control Computer Training Simulator
}

\author{
Zhou Quanzhi, a $^{1,}$ Qiu Chuanfei ${ }^{1, b}$, Cheng Huihua ${ }^{1, c}$, Nie Huiyu ${ }^{1, d}$, Tian \\ Weiqing ${ }^{1, d}$ and Li Jin ${ }^{1, d}$ \\ ${ }^{1}$ Wuhan Mechanical Technology College, Wuhan, Hubei, China \\ a1224225295@qq.com, b83950280@qq.com, cdugufei_2000@163.com, ${ }^{d} 416087321 @ q q . c o m$
}

Keywords: Job control. Vision. Simulation environment. Training scenarios.

Abstract. In the operation of the control job vehicle of a certain type of job control computer, with a big volume, the job vehicle is complex to operate and moreover, the operational training belongs to security-critical activities, which cannot be carried out indoors. Using computer training simulator to set up and control operational training, a training simulator of this type of job control computer is developed, through the job operation visual system to carry out job action, response and environmental simulation, which solves a series of problems encountered in the training process.

\section{Introduction}

A job control computer is the control hub of a certain job system, its small space can only accommodate one person inside, while there are more control objects which has a higher requirements in operating security. So, many function operations are not available under indoor training conditions. Therefore, in teaching and training, there are many restrictions from different aspects in the teaching and training of this job control computer, it is difficult to guarantee the completion of teaching towards multiple persons for multiple times within a very limited teaching time, resulting in low training efficiency, at the same time the training effect cannot be ensured. To solve this problem, this paper designed a set of job control computer training simulator, at the time of immersive virtual training environment to experience the training effects, the physical simulation can be operated. Compared with the relatively low cost of training, not only the problem of limited training space is solved, but also the training effect is improved. Simultaneous participation of many people in training improved the training efficiency, the immersive training environment greatly stimulated the training interest of students.

\section{System Design}

System Function Design. The original job control computer is embedded inside the vehicle, detecting working status of the job vehicle, and controlling the job vehicle to operate working. Its function block diagram is shown in Fig. 1.

Since the indoors teaching and training cannot complete the operation training of positioning systems, inertial navigation systems and operating control system, the development of training simulators must be compensated by training simulators. The job control computer training simulator has the functions of job operating, control input, status indicator, positioning operation, inertial navigation operation, hydraulic control operation, status detecting operation and other functions. Among them, the job operating function is to drive the job to work.

For compensating these trainings which cannot be carried out indoors through the training simulators, the function block diagram of this job control computer training simulator is shown in Fig. 2 .

System Structure Design. To achieve the above functions of job control computer training simulator, this job control computer training simulator consists of job visual computer and job control computer training simulator. Among them, the job visual computer mainly provides training environment for job control computer simulator, including training scenarios and the information 
environment required by job control computer simulator' running, while responding to control instructions issued by job control computer simulator and carrying out operations in training.

Job control computer simulator mainly provides computer the set of various parameters and job control training, issues control instructions to the job visual computer software, controls the operation of job vehicle in 3D training scenarios. Except that driving of job vehicle is performed by trained personnel through the job visual computer software, the remaining job operation depends on the job control computer simulator to complete.

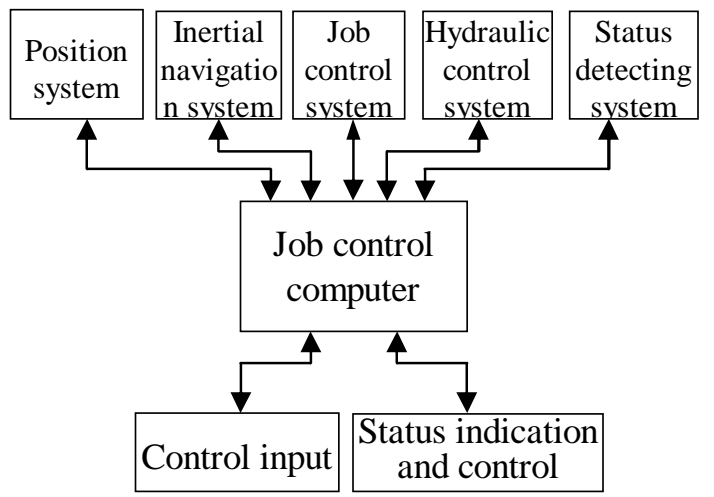

Fig. 1 Function block diagram of job control computer

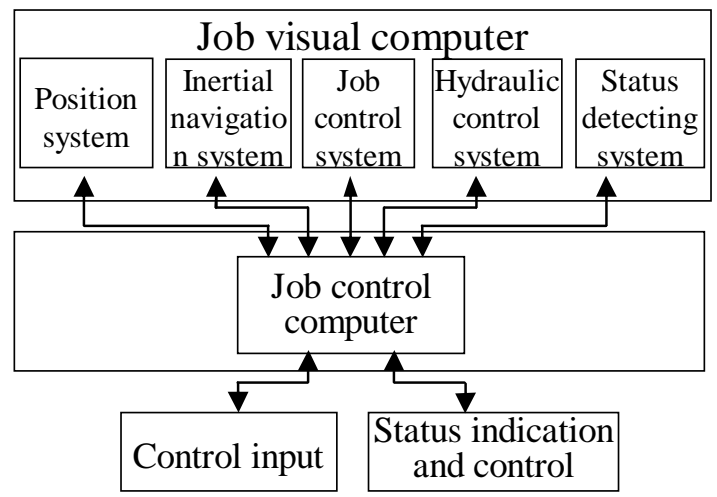

Fig. 2 Function block diagram of job control computer training simulator

\section{Hardware Design}

Hardware refers the development and design of job control computer simulator, which consists of the main board of job control computer, power panels, LCD screen and keyboard board, communication interface and other components, the structure is shown in Fig. 3.

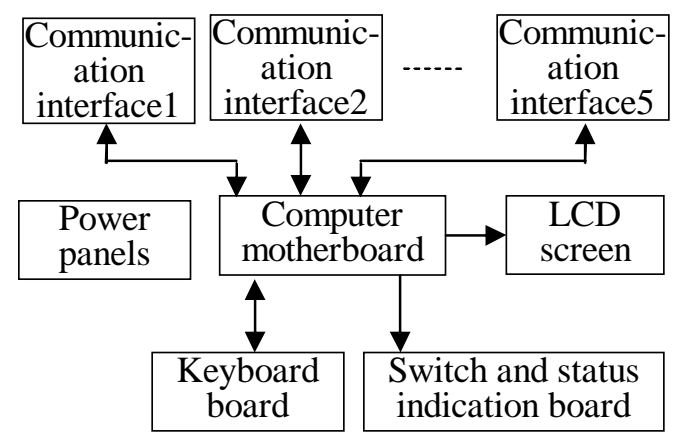

Fig.3 Hardware structure of job control computer simulator

Among them, the keyboard plate interconnects with computer through PS/2, completing the interconnection between the customized membrane keyboard and the computer's motherboard. Communication interface uses RS-232C standard interface. Through the input and output of computer's motherboard GPIO interfaces, the switch and status LED board detects the switch input, and controls the light on and off of status indicator as the vehicle working status indication. The power supply board adopts DC to ATX program, that is, convert power supplied to DC24V into ATX interfaces $3.3 \mathrm{~V}, \pm 5 \mathrm{~V}$ and $12 \mathrm{~V}$, at the same time provide SB5V and PSON, P.G functions to meet the power supply needs of motherboard[1], maintaining the DC power supply features of original job control computer.

\section{Software Design}

Software system mainly consists of job control computer simulator software and job visual computer software system.

Job Control Computer Simulator Software Design. The software mainly works according to the menus manner of the original software, realizing the interaction and communication with the job visual 
computer software system. The coordinate position in the scene of job vehicle and working status of job vehicle can be got from visual computer software, control command will be transmitted to job vehicle of job visual computer software to drive their job to work. It mainly consists of the menu system, main control module, communication module, database, data processing and keyboard HOOK and other modules, the structure is shown in Fig. 4.

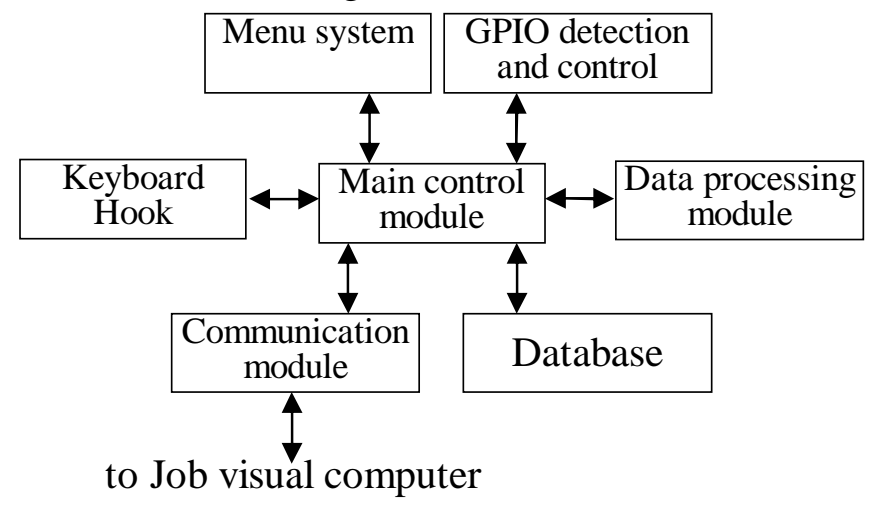

Fig. 4 Job control computer simulator software architecture diagram

Among them, the menu system is the operation input interface, keyboard HOOK is primarily responsible for intercepting the keyboard message transmission, and changing the keyboard input key values of a non-standard to its keyboard membrane of the same value labels, using system HOOK to implement key value change. Master module completes the control and coordination of the entire software, communication module uses controls multiple serial control to implement the data transmission used to position with job visual computer software. GPIO detection and control module mainly through its driver to proceed real-time read and write control to GPIO port, realizing the input of user switch operation and the output of state control. Data processing module solves, analyzes and processes the data required for the major storage operations.

Job Visual Computer Software System Design. Job visual computer software mainly simulates detection and control of training environment and job control computer. It uses Vtree virtual reality system job development platform and operating platform, mainly consisting of system control module, communication module, coordinate systems, 3D scenes and other modules, the structure is shown in Fig. 5.

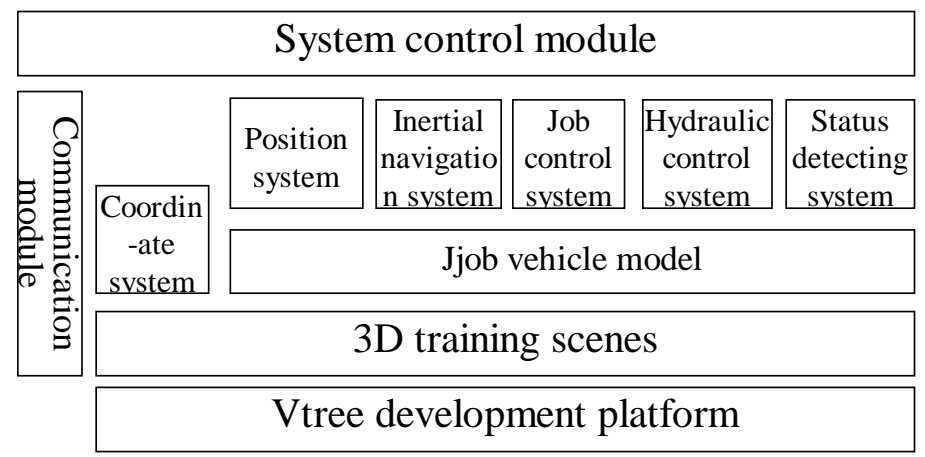

Fig. 5 Job visual computer software system structure

Wherein, Vtree virtual simulation platform can provide a wealth of SDK development kit during the development phase for integrating training scenarios and simulation solid model[2], completing the realization of motion control, fine motor control and presentation of each simulation. In the operational phase, it provides runtime environment for the advanced simulation, the screen rendering used in the virtual simulation the. 3D training scenarios utilizes the three-dimensional map developed by3D technology, natural environment and activity scenes. This coordinate system gives a set of coordinate data for 3D training scenarios, so that this three-dimensional map will have the same geographical coordinates and space systems with the real environment, in order to provide positioning data for the coordinate system and inertial navigation system. The coordinate origin of this coordinate system can 
be set and modified by the control module to avoid the data of each train are the same. Working vehicle models and 3D training scenarios are similar, the difference is that 3D training scenarios are stationary, requiring matching coordinate system, while working vehicle model is in motion, and a plurality of its subsidiary components are also motive, its movements are coordinated by the system control module and Vtree development platform[3].

The communication module is similar to the operation control computer simulator software communication module, and to achieve interoperability between the two. Positioning system will work in a 3D car model position to obtain the training scene coordinate system coordinates extracted coordinate display and control computer simulator based on the job requirements, the transmission through the communication module to the operations control computer simulator. Inertial navigation system and positioning system is similar, the difference lies in that the positioning system obtains absolute coordinates values from the coordinate system, while inertial navigation system require binding initial coordinates. Job vehicle calculates its current location according to the initial coordinates, belonging to a relative coordinate values. In general, in these two coordinate values, only one can be used to avoid the inherent error between the two coordinate values, resulting in the coordinate values of working vehicle unmatched.

Job control system and hydraulic control systems drive different parts of working vehicle to move, this motion is issued by the main control module of job control computer simulator via the communication module according to user operation. Job vehicle operation status and its own status are detected by the status detection systems, transmitted to job control computer simulator software via the communication module. To avoid the status detecting data provided by each training are the same, except coordinating and controlling the entire software system to run, but also the main control module can setup the case, that is, working vehicle status can be changed. This setting only affects the data of job control computer simulator provided by state detection system, but also affects the working action of job vehicle. For instance, if there is something wrong with the hydraulic system, then the job action related to job vehicle will be affected as well.

\section{Summary}

Through the above functional design, structural design, and hardware and software design, a set of job vehicle operation and training was constructed in this paper which cannot be completed in the room originally and trained more people in a group to carry out work simultaneously. A series of problems encountered on training have been solved perfectly by utilizing the job control computer simulator to achieve physical simulation, and by the means of combining with visual computer software virtual simulation. As a training subject, physical simulation makes the training more direct and effective, while virtual simulation provides required training environment of job vehicle, including the natural environment, information environment and operational feedback to improve the training experience in immersive way.

\section{References}

[1] Li Wengbing, The comprising theory of computer, forth ed., Tsinghua University Press, Beijing, 2010, pp. 201-203.

[2] Xu Zhi, Tang Shuo, Yan Xiaodong, Application of High Fidelity Large-Scale Terrain Database In Vtree, Computer Simulation. 23 (2006) 47-52.

[3] Guo Qisheng, Xie Wei, Real-Time Simulation Technology for Battlefield Vision, Journal of System Simulation, 12 (2013) 61-64. 